\title{
Average Room Occupancy Rate and its relation with Received Signal Strength Indicator in Wireless Sensors Networks
}

\author{
Daniel B. Barros, Alexandre Mota and Lia Mota
}

\begin{abstract}
This work aims to define a model for the relation between the Received Signal Strength Indicator (RSSI) of an Wireless Sensors Network (WSN) and the occupation rate of an indoor ambient. Experimental tests were carried out to identify this model that is able to provide the number of people (occupancy rate) in this ambient as a function of the RSSI variance. The tests were based on the installation of an WSN in a classroom, allowing to collect data related to RSSI variation, considering a point-to-point network. As a conclusion, it was possible to identify a relationship between these variables that determines the ambient occupancy rate.
\end{abstract}

Index Terms - Wireless Sensors Network, RSSI, Occupation
Rate

\section{INTRODUCTION}

$\mathrm{R}$ ECENTLY, there was a great technological advance in electronics industry that made possible to use wireless sensors networks, which are subject for research in various applications. With the characteristics of low cost and easy use these networks allow useful monitoring and security applications in critical locations, as well as the substitution of hazardous human work [1].

In this context, this work has the objective of identifying a mathematical model that represents the relation between the RSSI of an WSN and the occupancy rate (number of people) in an indoor ambient.

This work is organized as follows. Section II presents the main concepts of WSNs. Section III presents some variables and phenomena that have influence in the radio signal propagation and, consequently, on RSSI. Section IV brings some concepts about occupancy rate. Section V describes the methodology adopted in this work and Section VI presents the obtained results. Finally, Section VII presents the conclusions of this work.

\section{WIRELESS SENSORS NETWORKS (WSNS)}

A wireless sensors network is someway dedicated to a

Daniel B. Barros, Alexandre Mota and Lia Mota are with the Electrical Engineering Faculty, Pontifical Catholic University of Campinas, Rod. Dom Pedro I, Km 136, CEP 13086-900, Brazil (e-mails: danielbragabarros@gmail.com, amota@puc-campinas.edu.br, lia.mota@puccampinas.edu.br). specific application. It is composed by one or more transceiver nodes with autonomous capacity of data acquisition and processing [1]. The nodes transmission characteristics and the reception sensibility limit its distribution on the ambient, affecting their communication reliability. Figure 1 shows a wireless sensors network with point-to-point topology, in which each node communicates directly with the base.

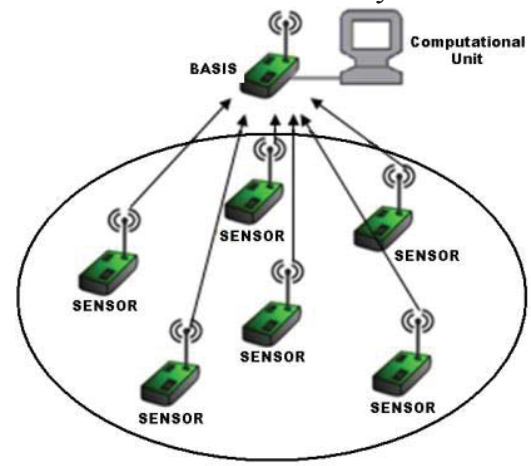

Fig.1. Wireless Sensors Network configuration example.

Many researches were made in WSNs showing their technological benefits, like reduction of energy consumption, lowering costs, network devices size and the functional capacity raise [2]. Wireless Sensors Networks are current being applied in vehicles, intelligent networks, intelligent buildings and intelligent cities. These networks can also be used in medical, environmental, military and commercial areas. Because of their wireless nature, these networks can easily measure the radio signal characteristics from the ambient, like received power from each one of the other network nodes [5]. This feature is detailed in the next section.

\section{ReCEIVED Signal StRENGTH}

The Received Signal Strength Indicator (RSSI) can be defined as the power that a device (which composed the WSN) received from the radio infrastructure (WSN) at a given ambient. The RSSI is directly influenced by the phenomena and variables described as follows.

\section{A. Propagation}

Radio signals are vulnerable to propagation phenomena, which can change signals' characteristics, such as attenuation, reflection, refraction, diffraction, dispersion and noise [3]. The result of each of these phenomena directly impacts the RSSI 
value.

Integrated circuits designed for sensors are capable of acquiring RSSI measurements. This value is estimated from the received power level converted in $\mathrm{dBm}$ when reading from the circuit registers [4]. There are many areas for research in this application field like sensors positioning, propagation medium, hardware performance, and others [1]

\section{B. Environment influence}

Radio signal propagation can be also affected by environment changes. In indoor ambient, the objects inside the room become obstacles to the radio signal propagation and so they disturb the signal.

Network nodes can monitor the RSSI, making possible to know the signal strength between network nodes in an indoor environment [3].

\section{People influence}

In an indoor environment people are doing their daily activities, and they also can be considered obstacles to radio signals propagation. In this way, one can consider the same observations related to objects.

\section{OCCUPANCY RATE}

The term "Occupancy Rate" is used in different situations. For example, in Telecommunications it can represent the occupation of a telephonic channel; in Civil Engineering this term refers to the built area in relation to the total ground area. This work proposes a study about the feasibility of using an WSN to estimate the occupancy rate (number of people) in an indoor ambient, based on RSSI measurements.

This work motivation is related to the possibility of determining the occupancy rate in a non-invasive and low cost way with wireless devices.

\section{Methodology}

An experimental approach was chosen to verify this work proposition. For this reason, a standard classroom (at the Pontifical Catholic University of Campinas - PUC-Campinas) was chosen to host the experiments. It was populated with a simple wireless sensors network consisting of one sensor communicating with one base with direct line of sight and a height that allows people to interfere in the signal. The network topology and used equipment are shown in Figure 2.

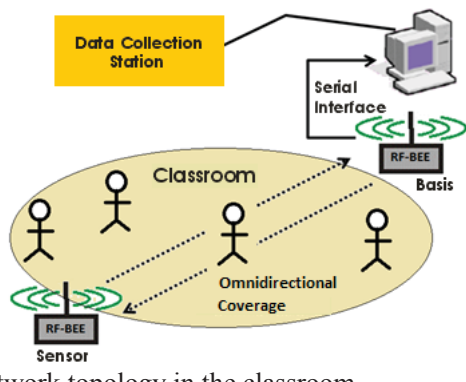

Fig. 2. Test network topology in the classroom.

The classroom used has about $54 \mathrm{~m}^{2}$ and $3,5 \mathrm{~m}$ height. The nodes were installed in two parallel walls, $6 \mathrm{~m}$ from each other and at $1,3 \mathrm{~m}$ from the floor. This allows people passing to change the characteristic of the wireless communication.

RFBee device [6] was used as sensor and base nodes. RFBee is composed by CC1101 transceiver and ATMega-328 microcontroller [6]. This architecture was chosen for its simplicity, widespread and Arduino compatibility, using C programming language. It is important to note that this node was not feed by batteries and, so, didn't presented problems with short lifetime, because the sensor node was fed by AC power source.

Using the pooling strategy, where the base requests the sensor a measurement, the base and sensor were programmed with the Radiuino platform code [6], allowing users to easily program and run a wireless sensors network.

The sensor node was fed by AC power source, ensuring data collecting. The sensor's main function is to receive a requisition from the base and retransmit it back. The data itself is not important, for we are interested in the RSSI only. The returned packet contains the signal strength in its first four bytes, allowing the base to know the RSSI of the uplink and downlink transactions.

The base node is connected to the computer by a USB cable and transfers data to proprietary software that makes requisitions in fixed time intervals and saves the RSSI data into a file for analysis and configured to request measurements at every 30 seconds. This time interval was considered a good start point to measure people coming and going in the environment and also friendly to the storage available capacity in the computer. The real people count in the classroom was given by the teacher's grade book.

This test was carried out during a week, recording the RSSI for every class, break, lunch and night times. Post processing of data was done to choose time intervals of interest, like a full class, half class, night time, and others. The signal variance was adopted as a variable in order to investigate the relationship between RSSI and the room occupancy rate.

\section{Results}

The obtained results are presented by graphics that show the RSSI evolution during a time interval. These graphics (one for each "occupancy rate" condition) are shown in Figure 3.
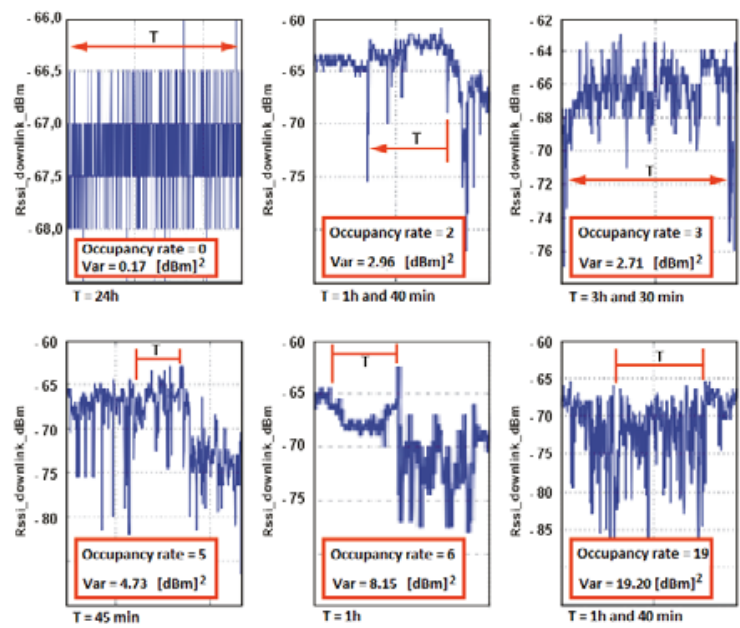

Fig. 3. RSSI variation as a function of time, in the presence of $0,2,3,5,6$ and 19 people. 
It is possible to represent the RSSI variance as a function of the occupancy rate, based on the values presented in Figure 3, as can be seen in Figure 4.

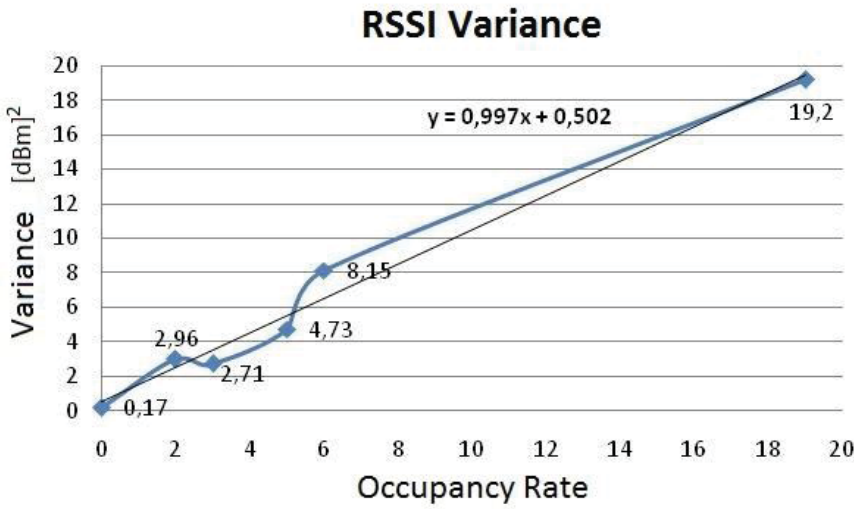

Fig. 4. Signal variance as a function of the occupancy rate and trend curve.

Figure 4 shows a linear variance trend curve, which can be expressed by the mathematical model defined by Equation 1.

$y=0,9973 . x+0,5027$

Where $\mathrm{x}$ represents the occupancy rate and $\mathrm{y}$ represents the variance of the variable $\mathrm{x}$.

A different time interval was selected to validate the identified mathematical model. In this interval, there were 3 people in the ambient, keeping the variance in 3,17 , as shown in Figure 5.

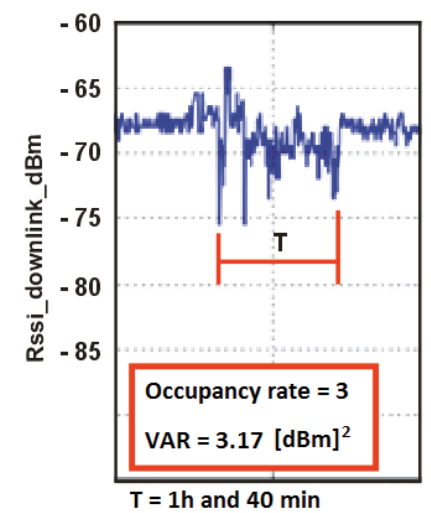

Fig. 5. Variance as a function of time in the presence of 3 people (occupancy rate equals to 3 ).

When the $\mathrm{x}$ variable was isolated in Equation 1, it was possible to replace the variance value $(3,17)$ of the new interval, verifying the expected occupancy rate (3), as follows.

$$
\begin{aligned}
& x=\frac{(y-0,5027)}{0,9973} \\
& x=\frac{(3,17-0,5027)}{0,9973} \\
& x=2,67
\end{aligned}
$$

It can be seen that the estimated occupancy rate $(2,67)$ is approximately equal to the expected value (3). This number is compatible with the real number of people in the ambient in the time of the current measurement.

\section{CONCLUSION}

From the experiments that were carried out, one conclude that it is possible to find a relationship between an indoor ambient occupancy rate and the wireless sensors network RSSI variance. It is relevant to consider that, when dealing with people, the predicted values always have to be an integer, that's why the error has to be calculated and the value rounded.

The methods used to calculate the occupancy rate can be improved, for example, taking a better control over people number in the room and leading to a precise definition of the trend curve's coefficients.

This work used only IEEE 802.15.4 sensors, but in future studies other technologies can be tested, like the IEEE 802.11 which are more used in buildings and with potential for other applications.

\section{ACKNOWLEDGMENT}

The authors would like to thank the Pontifical Catholic University of Campinas (PUC-Campinas) for the support to this work.

\section{REFERENCES}

[1] G. Gracioli, et al. "Evaluation of an RSSI-based Location Algorithm for Wireless Sensor Networks," IEEE Latin America, vol. 9, pp. 830-835, Aug., 2013.

[2] J. C. A. Cansado, "Instrumentalization of Wireless Sensor Networks," Ph.D. dissertation, Electrical Engineering Dept., USP, São Paulo, SP, Brazil, 2011.

[3] E. T. Procópio, "Identification of a mathematicaç model to represent wireless sensors networks' transmission in buildings," M.S. thesis, Electrical Engineering Dept., PUC-Campinas, Campinas, SP, Brazil, 2012

[4] Texas Instrumments, CC1101 Low-power Sub-1 GHz RF Transceiver, 2011

[5] G. Kiev, A. Alvaro, E.C. Peixoto. "Towards a Technological Maturity Model for Smart Cities," in SBS., São Paulo, SP, 2012.

[6] Radiuino website. Available online: http://www.radiuino.cc (accessed on $04012015)$. 